\title{
Valence state of Sm in single crystalline EuO thin films
}

\author{
A. Reisner, ${ }^{1}$ D. Kasinathan, ${ }^{1}$ S. Wirth,${ }^{1}$ L. H. Tjeng, ${ }^{1}$ and S. G. Altendorf ${ }^{1}$ \\ ${ }^{1}$ Max Planck Institute for Chemical Physics of Solids, Nöthnitzer Str. 40, 01187 Dresden, Germany
}

\begin{abstract}
Samarium has two stable valence states, $2+$ and $3+$, which coexist in many compounds forming spatially homogeneous intermediate valence states. We study the valence state of samarium when incorporated in a single crystalline $\mathrm{EuO}$ thin film which crystallizes in a $f c c$-structure similar to that of the intermediate valence $\mathrm{SmO}$, but with a larger lattice constant. Due to the increased lattice spacing, a stabilization of the larger $\mathrm{Sm}^{2+}$ ion is expected. Surprisingly, the samarium incorporated in $\mathrm{Sm}_{\mathrm{x}} \mathrm{Eu}_{1-\mathrm{x}} \mathrm{O}$ thin films shows a predominantly trivalent character, as determined by $\mathrm{x}$-ray photoelectron spectroscopy and magnetometry measurements. We infer that the $\mathrm{O}^{2-}$ ions in the EuO lattice have enough room to move locally, so as to reduce the Sm-O distance and stabilize the $\mathrm{Sm}^{3+}$ valence. (Dated: April 06, 2017)
\end{abstract}

\section{INTRODUCTION}

Interest in the study of samarium containing compounds has increased recently, owing to the theoretical prediction of topologically nontrivial surface states in $\mathrm{SmB}_{6}[1,2]$, the high-pressure golden phase of $\mathrm{SmS}[3]$, and ambient $\mathrm{SmO}$ [4]. The speciality of samarium arises from the perpetual competition between its two stable valence states: nonmagnetic $\mathrm{Sm}^{2+}$ and a magnetic $\mathrm{Sm}^{3+}$, which is stabilized in many solids by the formation of spatially homogenous non-integral intermediate valence $\mathrm{Sm}^{2 \cdot \mathrm{x}+}$. Experimental examples of samarium containing intermediate valence compounds include the extensively studied $\mathrm{SmB}_{6}[5,6]$; high-pressure golden-phases of $\mathrm{SmS}, \mathrm{SmSe}$ and SmTe [7]; ambient $\mathrm{SmO}$ [8]; $\mathrm{SmFe}_{2} \mathrm{Al}_{10}$ [9]; $\mathrm{Sm}_{2.75} \mathrm{C}_{60}$ [10]; and elemental samarium metal itself [11]. The intermediate valence state of samarium can be understood as an intra-atomic promotion of $4 f$ electrons to $5 d$ bands, going from $\mathrm{Sm}^{2+}\left(4 f^{6} 5 d^{0}\right)$ to $\mathrm{Sm}^{3+}$ $\left(4 f^{5} 5 d^{1}\right)$. Combined with the effects of strong electronic correlations and strong spin-orbit coupling, various exotic ground states are realizable in samarium based materials.

In this work we select the $\mathrm{SmO}$ and $\mathrm{EuO}$ system and address the question of the valence of Sm in single crystalline $\mathrm{EuO}$ films. Our interest is motivated by the proposal [4] that $\mathrm{SmO}$ is a candidate material for having topologically protected surface states and that a thin film interface of $\mathrm{SmO}$ with the ferromagnetic semiconductor EuO may make an excellent contender towards realizing the quantum anomalous Hall effect in strongly correlated electron systems. While the preparation of high quality single crystalline EuO thin films is well established [12], no report exists to our knowledge about SmO films. In fact, the synthesis of $\mathrm{SmO}$ as bulk material requires high pressures $[13,14]$, suggesting that the preparation of $\mathrm{SmO}$ in thin film form may not be possible using standard thin film deposition techniques. As a start, we are therefore interested to make a system containing $\mathrm{SmO}_{6}$ octahedral units by utilizing $\mathrm{EuO}$ as the stabilizing host material, i.e. to prepare $\mathrm{Sm}_{\mathrm{x}} \mathrm{Eu}_{1-\mathrm{x}} \mathrm{O}$, and study the local properties of the $\mathrm{Sm}$.

Both $\mathrm{SmO}$ and EuO crystallize in a face centered cubic
( $f c c$ ) lattice with comparable lattice constants of 4.9414 $\AA$ [14] and 5.1435 $\AA$ [15], respectively. Based on empirical estimates of the lattice constants for $\mathrm{Sm}^{3+} \mathrm{O}(4.917$ $\AA)$ and $\mathrm{Sm}^{2+} \mathrm{O}(5.15 \AA),[13]$ the samarium valency in bulk $\mathrm{SmO}$ was determined to be $2.9+$, not inconsistent with x-ray absorption spectroscopy measurements [14]. $\mathrm{EuO}$ on the other hand consists of the integer valent and magnetic $\mathrm{Eu}^{2+}$, and exhibits many spectacular properties including a nearly $100 \%$ spin polarized conduction band [16, 17], a metal-to-insulator transition (MIT) [18] and a colossal magneto-resistance [19] in the slightly Eu-rich compound, as well as large magneto-optical effects [20, 21]. We utilize the well established Eu distillation technique [12] to grow high-quality single crystalline $\mathrm{Sm}_{\mathrm{x}} \mathrm{Eu}_{1-\mathrm{x}} \mathrm{O}$ thin films by molecular beam epitaxy (MBE) under ultra high vacuum conditions. We expect that the larger $\mathrm{EuO}$ lattice will allow the $\mathrm{Sm}$ to be stabilized in the $2+$ valence state or at least in an intermediate valence state closer to $2+$ compared to bulk SmO.

\section{EXPERIMENTAL METHODS}

Thin films of $\mathrm{Sm}_{\mathrm{x}} \mathrm{Eu}_{1-\mathrm{x}} \mathrm{O}$ were grown in a MBE system with a base pressure of $1 \times 10^{-10}$ mbar. Prior to growth, epi-polished yttria stabilized zirconia (YSZ) substrates with a (001) surface (provided by CrysTec $\mathrm{GmbH}$ ) were annealed for two hours at $600{ }^{\circ} \mathrm{C}$ in an oxygen atmosphere of $5 \times 10^{-7}$ mbar. High purity Eu metal (Ames Laboratory) and Sm metal (Hunan HMC Metals C., Ltd.) were co-evaporated from effusion cells (LUXEL RADAK I). The Sm was additionally purified by degassing at about $1000{ }^{\circ} \mathrm{C}$ in a Mo ampulla before use.

$\mathrm{Eu}$ was evaporated using a flux rate of $8.0 \pm 0.2 \AA / \mathrm{min}$ at temperatures of about $570{ }^{\circ} \mathrm{C}$. Sm was evaporated at flux rates of $0.02-1.5 \AA / \mathrm{min}$ at temperatures between $450-520{ }^{\circ} \mathrm{C}$. Molecular oxygen was supplied via a high precision leak valve and set to a partial pressure of about $3.0 \times 10^{-8}$ mbar during film growth. The YSZ substrates were heated to $400{ }^{\circ} \mathrm{C}$ during deposition. These growth parameters were calibrated to fulfill the 

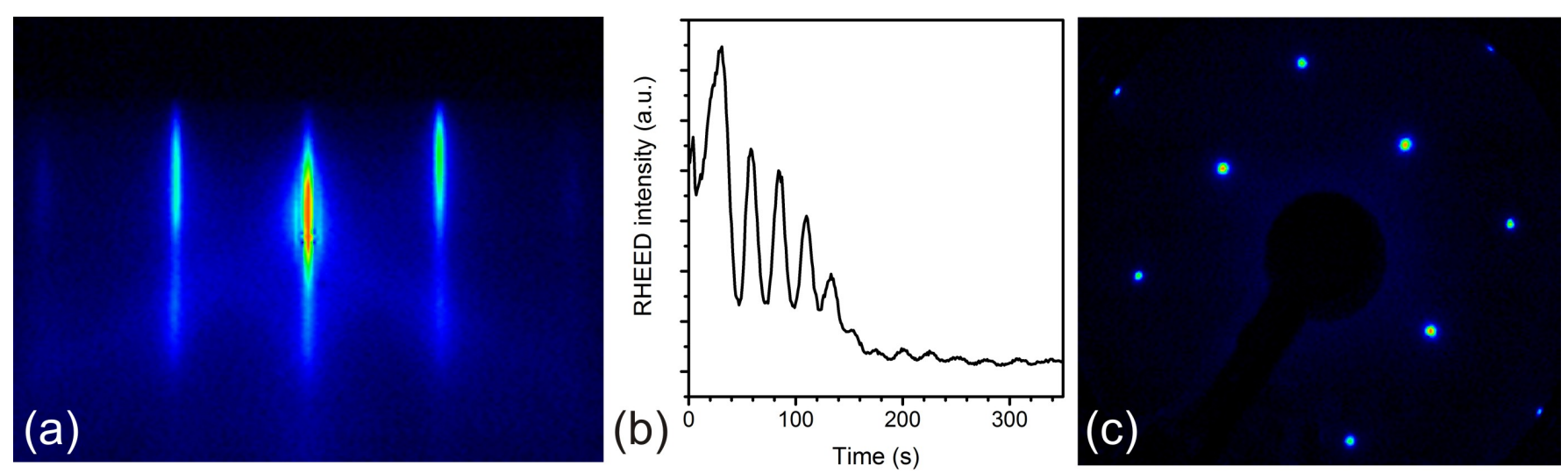

FIG. 1. (a) RHEED image of the EuO thin film with $9 \% \mathrm{Sm}$. (b) corresponding RHEED intensity oscillations of the specularly reflected electron beam. (c) LEED pattern of the EuO film with $9 \% \mathrm{Sm}$ recorded at $61 \mathrm{eV}$.

\begin{tabular}{|c|c||c|c|}
\hline \multicolumn{2}{|c||}{$\begin{array}{c}\text { Sm concentration }(\%) \\
\text { from XPS }\end{array}$} & \multicolumn{2}{c|}{ Thickness $(\mathrm{nm})$} \\
\hline- & 2.4 & 39 & 13 \\
5.2 & 5.2 & 38 & 11 \\
9.1 & 9.8 & 38 & 12 \\
12.0 & 11.9 & 43 & 12 \\
17.5 & 13.0 & 43 & 9 \\
19.9 & 20.0 & 43 & 10 \\
\hline
\end{tabular}

TABLE I. Overview of Sm concentrations as derived from $\mathrm{x}-$ ray photoelectron spectra and $\mathrm{Eu} / \mathrm{Sm}$ flux rates (see text), and layer thicknesses as deduced from x-ray reflectivity measurements. Cr was used for capping.

Eu distillation condition [12]. The growth was monitored by reflection high energy electron diffraction (RHEED) using a STAIB Instruments EK-35-R system. The diffraction patterns were recorded at an electron energy of $15 \mathrm{kV}$. After 60 minutes, the growth was terminated by first closing the oxygen supply and the Sm shutter at the same time. To avoid a formation of $\mathrm{Eu}^{3+}$ at the film surface, after further 30 seconds the $\mathrm{Eu}$ shutter was closed and the substrate cooled down to room temperature. Two reference films were additionally prepared: i) intermediate valence metallic $\mathrm{Sm}$ was deposited at room temperature onto an annealed YSZ substrate, ii) a trivalent $\mathrm{Sm}_{2} \mathrm{O}_{3}$ film was grown at room temperature onto a $\mathrm{Sm}$ film at a $\mathrm{Sm}$ evaporation rate of $7.1 \AA / \mathrm{min}$ in an oxygen partial pressure of $1 \times 10^{-7}$ mbar.

The films were analyzed in situ by x-ray photoelectron spectroscopy (XPS), utilizing a monochromatized $\mathrm{Al}$ $K_{\alpha}$ x-ray source $(\mathrm{h} \nu=1486.6 \mathrm{eV})$ and a Scienta R3000 electron energy analyzer. Spectra were collected at room temperature and in normal emission geometry. Subsequently, low energy electron diffraction (LEED) measurements were performed using a Thermo VG Scienta T191 system. For further ex situ characteri- zation, the films were covered with a capping layer of about $100 \AA$ thick $\mathrm{Cr}$ to prevent the sensitive $\mathrm{EuO}$ from degradation in air. X-ray diffraction (XRD), $\mathrm{x}$-ray reflectivity (XRR) measurements and reciprocal space maps (RSM) were recorded in an X'Pert PRO four circle diffractometer from PANalytical equipped with a monochromatized $\mathrm{Cu} K_{\alpha 1}$ radiation source and a PIXcel-3D detector. The magnetic properties were investigated using a Quantum Design MPMS XL5 magnetometer. The magnetization curves were measured by applying an in-plane magnetic field of 50 Oe during the temperature sweeps. Details of the samples - Sm concentrations and layer thicknesses - are summarized in table I.

\section{RESULTS}

The crystalline quality of the $\mathrm{Sm}_{\mathrm{x}} \mathrm{Eu}_{1-\mathrm{x}} \mathrm{O}$ thin films is confirmed by the sharp streaks of the RHEED patterns for the entire substitution range reported. An exemplary electron diffraction image of the EuO sample with $9 \%$ $\mathrm{Sm}$ is shown in fig. 1 (a). The intensity oscillations of the specularly reflected electron beam reveal a smooth layer-by-layer growth (fig. 1 (b)). One can distinguish two different growth mechanisms which are characteristic for the deposition of $\mathrm{EuO}$ on YSZ, cf. Ref. [12]. In the initial growth, oxygen is supplied mainly from the YSZ substrate yielding the first pronounced intensity oscillations typical for the growth of $\mathrm{EuO}$ on YSZ. After the completion of five monolayers, the oxygen is supplied only through the partial oxygen pressure in the growth chamber. The LEED pattern in fig. 1 (c) reflects the fourfold symmetry of the (001) facet of the $f c c$ rock salt structure and indicates a well ordered single crystalline surface.

The structure of the $\mathrm{Sm}_{\mathrm{x}} \mathrm{Eu}_{1-\mathrm{x}} \mathrm{O}$ thin films was analyzed in more detail by ex situ XRD measurements (fig. 2 ). The $\theta-2 \theta$ scans show the sharp (002) peaks of the 

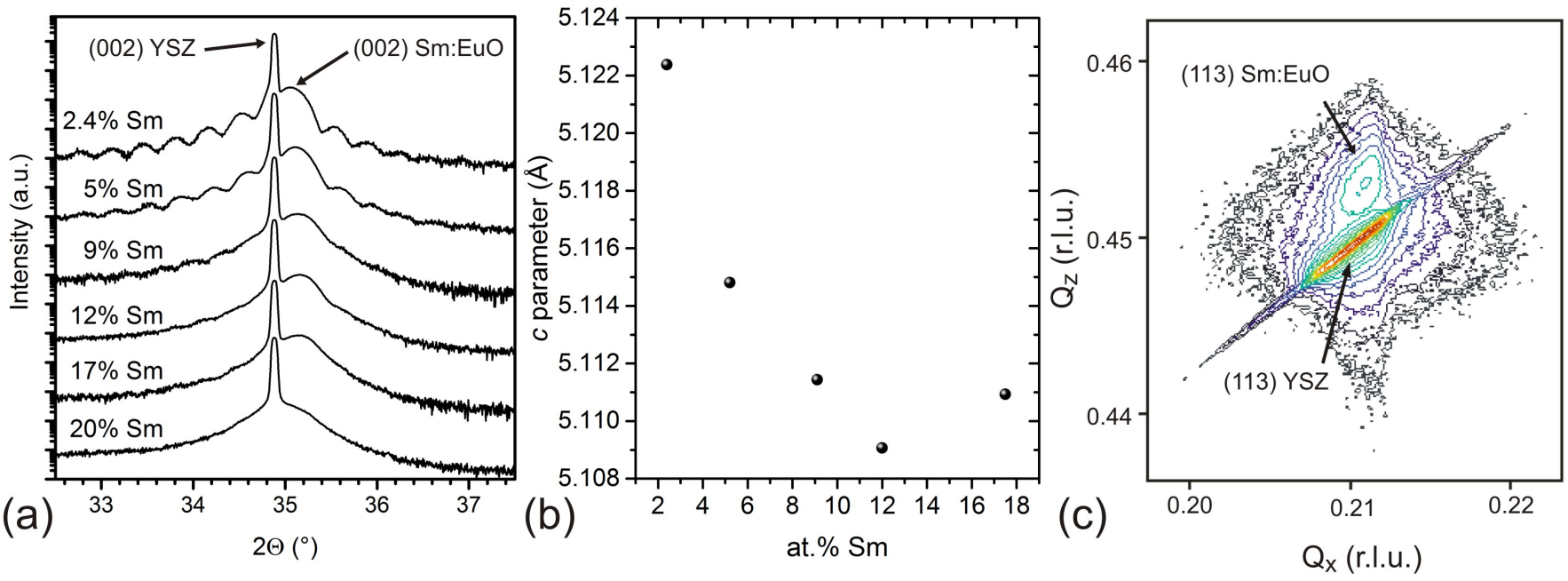

FIG. 2. (a) $\theta-2 \theta$ XRD scans around the (002) reflection of the $\mathrm{Sm}_{\mathrm{x}} \mathrm{Eu}_{1-\mathrm{x}} \mathrm{O}$ films. (b) The corresponding out-of-plane (c) lattice parameters calculated from the $2 \theta$ value as a function of Sm concentration. (c) XRD reciprocal space map around the (113) reflection of the $\mathrm{EuO}$ film with $17 \% \mathrm{Sm}$.

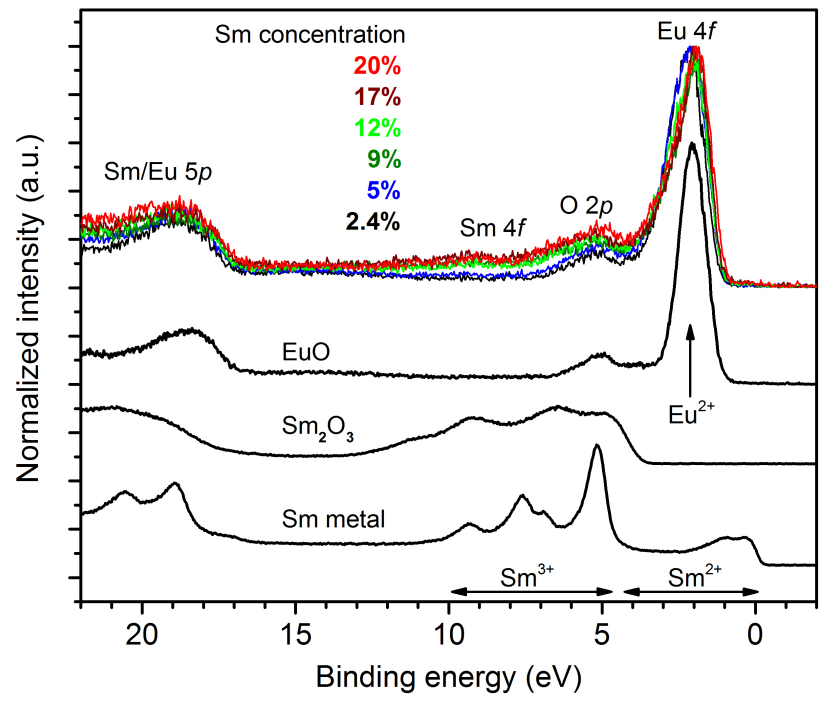

FIG. 3. XPS spectra of the valence band region of the $\mathrm{Sm}_{\mathrm{x}} \mathrm{Eu}_{1-\mathrm{x}} \mathrm{O}$ thin films for various $\mathrm{Sm}$ concentrations up to $20 \%$. For comparison, the spectra of a pure $\mathrm{EuO}$ film, a $\mathrm{Sm}_{2} \mathrm{O}_{3}$ thin film and a pure $\mathrm{Sm}$ metal film are shown additionally. The contributions of $\mathrm{Sm}^{3+}$ and $\mathrm{Sm}^{2+}$ to the intermediate valence $\mathrm{Sm}$ metal spectrum are indicated. [22]

YSZ substrate and, at slightly higher diffraction angles, the broader (002) peaks of the $\mathrm{Sm}_{\mathrm{x}} \mathrm{Eu}_{1-\mathrm{x}} \mathrm{O}$ films with interference fringes, a signature of the finite layer thickness. With increasing Sm substitution concentration, the film peaks shift to larger $2 \theta$ diffraction angles, i.e., the out-of-plane $(c)$ lattice constant decreases. The deduced lattice parameters are summarized in fig. 2 (b). The thickness fringes vanish for high Sm substitution concentrations indicating an increasing disorder and roughness. The RSM analysis reveals that the in-plane lattice pa- rameter of $\mathrm{Sm}_{\mathrm{x}} \mathrm{Eu}_{1-\mathrm{x}} \mathrm{O}$ adapts to the YSZ substrate such that the grown films are coherently strained up to a Sm substitution level of $17 \%$, see fig. 2 (c). This is not inconsistent with the reported solubility of 14 at.\% Sm in $\mathrm{EuO}$ bulk samples [23]. In fact, our observation that the out-of-plane lattice constant of the $17 \%$ sample does not follow the decreasing trend may indeed indicate that we have exceeded the solubility here.

To check the chemical composition and the valence states of the constituents, in situ XPS measurements were performed. The valence of the $\mathrm{Eu}$ atoms in $\mathrm{Sm}_{\mathrm{x}} \mathrm{Eu}_{1-\mathrm{x}} \mathrm{O}$ films can be directly deduced from the valence band scans shown in fig. 3. The $4 f^{7} \rightarrow 4 f^{6}$ transition at about $2 \mathrm{eV}$ binding energy corresponds to the $\mathrm{Eu}^{2+}$ valence state. The absence of the $\mathrm{Eu}^{3+}$ peak at $7 \mathrm{eV}$ confirms the purely divalent character of the Eu. With increasing Sm content, the Sm $4 f$ states can be recognized in the range of $4-10 \mathrm{eV}$ binding energy. A comparison with the trivalent $\mathrm{Sm}_{2} \mathrm{O}_{3}$ reference indicates that the $\mathrm{Sm}$ valence in the films is predominantly $\mathrm{Sm}^{3+}$. In fig. 4, we have simulated the spectrum of $\mathrm{Sm}_{0.12} \mathrm{Eu}_{0.88} \mathrm{O}$ by composing a spectrum consisting of $12 \%$ trivalent $\mathrm{Sm}_{2} \mathrm{O}_{3}$ and $88 \%$ divalent $\mathrm{EuO}$. The spectral weight around $5-10 \mathrm{eV}$ binding energy is reproduced quite well, implying the trivalent nature of the incorporated Sm.

The verification of possible $\mathrm{Sm}^{2+} 4 f$ states in the valence band region is rather difficult due to the intense $\mathrm{Eu}^{2+}$ peak at $2 \mathrm{eV}$ binding energy. For a clear detection of $\mathrm{Sm}^{2+}$, therefore, the Eu $3 d$ and Sm $3 d$ core levels were measured. Figure 5 exhibits the core level spectra with the integral background [24] subtracted. The integrated intensity of the peaks has been used to determine the Sm content of $\mathrm{Sm}_{\mathrm{x}} \mathrm{Eu}_{1-\mathrm{x}} \mathrm{O}$ films, see table I. The resulting Eu:Sm ratios fit in general reasonably well with the 


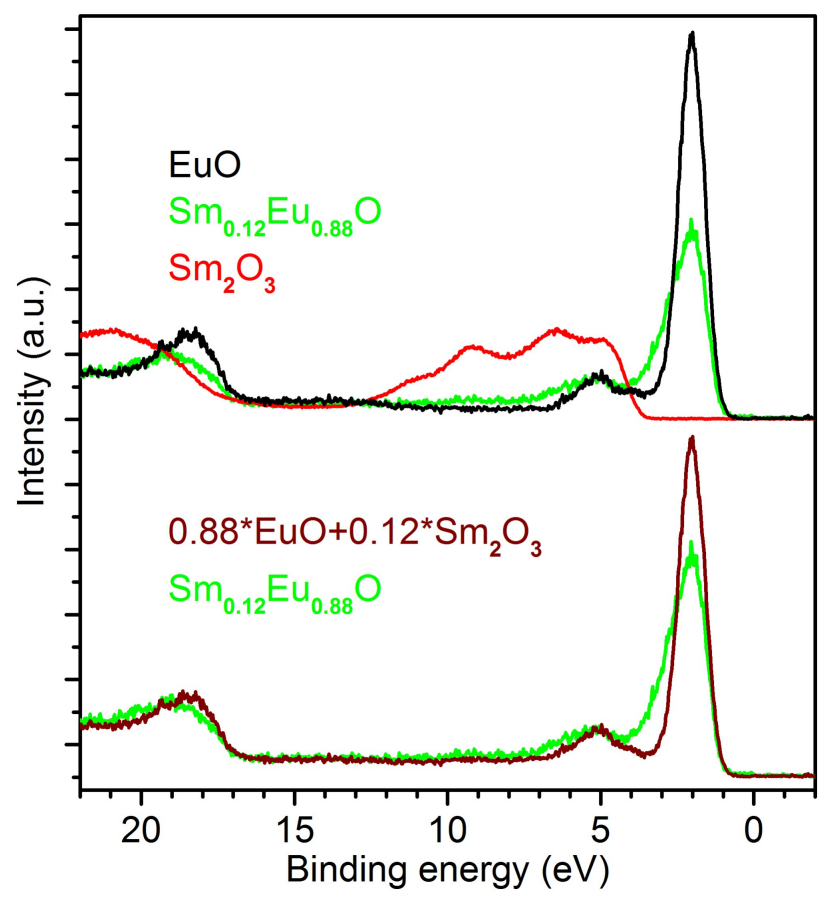

FIG. 4. top: XPS spectra of the valence band region of the pure $\mathrm{EuO}$, the $\mathrm{EuO}$ with $12 \% \mathrm{Sm}$, and a $\mathrm{Sm}_{2} \mathrm{O}_{3}$ thin film. bottom: A comparison of a reference spectrum composed of $12 \%$ of the $\mathrm{Sm}_{2} \mathrm{O}_{3}$ spectrum and $88 \%$ of the $\mathrm{EuO}$ spectrum, and the measured spectrum of the EuO thin film with $12 \%$ Sm.

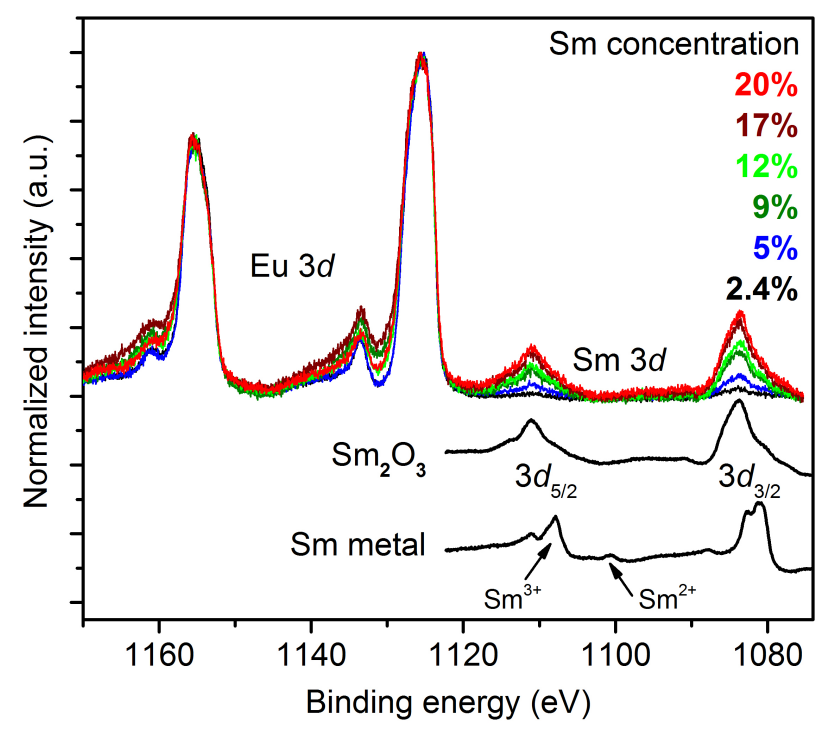

FIG. 5. XPS spectra of the Eu $3 d$ and Sm $3 d$ core level regions of the $\mathrm{Sm}_{\mathrm{x}} \mathrm{Eu}_{1-\mathrm{x}} \mathrm{O}$ thin films. For comparison the spectra of a $\mathrm{Sm}_{2} \mathrm{O}_{3}$ thin film and a pure $\mathrm{Sm}$ metal film are shown additionally. The energy position of the $\mathrm{Sm}^{3+}$ and $\mathrm{Sm}^{2+}$ contributions to the $3 d_{5 / 2}$ level of Sm metal are indicated. $[25]$ calculated ratios deduced from the metal fluxes measured using a quartz crystal microbalance and assuming identical re-evaporation rates of $\mathrm{Eu}$ and $\mathrm{Sm}$. We note that for the film with the lowest Sm concentration of 2.4 $\%$ the Sm 3d peak intensity is only of the order of the background. Therefore, this doping level was estimated solely based on the flux ratios. The shape of the Sm $3 d$ core levels in $\mathrm{Sm}_{\mathrm{x}} \mathrm{Eu}_{1-\mathrm{x}} \mathrm{O}$ matches well with the ones of the $\mathrm{Sm}_{2} \mathrm{O}_{3}$ reference confirming the predominantly trivalent state of $\mathrm{Sm}$ in our films. We do not observe any indication for the presence of metallic Sm or $\mathrm{Sm}^{2+}$ within the limits of the measurement resolution.

In the next step, we investigate the influence of the $\mathrm{Sm}$ on the magnetic properties of $\mathrm{EuO}$ to verify that the $\mathrm{Sm}^{3+}$ ions are successfully integrated into the $\mathrm{EuO}$ matrix as indicated by the structural analysis. The incorporation of trivalent dopants into the $\mathrm{EuO}$ lattice is known to enhance the relatively low Curie temperature $\left(T_{C}\right)$ of $69 \mathrm{~K}$ for the stoichiometric compound [26]. Examples include doping with trivalent rare earth ions like Gd [27-31], La [17, 27, 31, 32], Lu [31], and $\mathrm{Nd}[27]$ or trivalent transition metals ions like $\mathrm{Y}$ [27] and Sc [33]. Indeed, also our $\mathrm{Sm}_{\mathrm{x}} \mathrm{Eu}_{1-\mathrm{x}} \mathrm{O}$ films show enhanced Curie temperatures. The temperature dependent magnetization curves are presented in fig. 6 . The $\mathrm{EuO}$ reference sample shows the typical Brillouin like shape with a $T_{C}$ of $69 \mathrm{~K}$. Upon incorporation of $\mathrm{Sm}, T_{C}$ increases significantly with a maximum of 122 $\mathrm{K}$. The shape of the magnetization curves deviate from a Brillouin function (except perhaps for the $5 \%$ film), indicating the presence of phase separation, i.e., part of the film orders magnetically at $122 \mathrm{~K}$ and another part at $69 \mathrm{~K}$ or lower. The observed elevated Curie temperature of the $\mathrm{Sm}_{\mathrm{x}} \mathrm{Eu}_{1-\mathrm{x}} \mathrm{O}$ films proves the effective integration of samarium as dopant into the $\mathrm{EuO}$ lattice and also provides further evidence for the predominantly trivalent character of the incorporated $\mathrm{Sm}$. In the 1970s, a few studies about the substitution of $\mathrm{Eu}^{2+}$ by $\mathrm{Sm}$ in $\mathrm{EuO}$ polycrystalline samples $(\mathrm{Sm}: \mathrm{EuO})$ were published in which a trivalent character of the built-in Sm ions was reported [34], and a higher maximum Curie temperature of about $130 \mathrm{~K}$ was found $[23,35]$. The magnetization curves of the $\mathrm{Sm}_{\mathrm{x}} \mathrm{Eu}_{1-\mathrm{x}} \mathrm{O}$ films exhibit a striking similarity with $\mathrm{Gd}$-doped $\mathrm{EuO}$ which has a slightly higher maximum $\mathrm{T}_{C}$ of $125 \mathrm{~K}$ [30]. The $3 \mathrm{~K}$ lower $\mathrm{T}_{C}$ value of the Sm-doped films may be explained by the slightly larger ionic radius of $\mathrm{Sm}^{3+}$ compared to $\mathrm{Gd}^{3+}$, and thus a smaller compression of the lattice, consistent with our previous band structure calculations. $[36]$

Having unambiguously established that samarium is trivalent in $\mathrm{Sm}_{\mathrm{x}} \mathrm{Eu}_{1-\mathrm{x}} \mathrm{O}$, we now address the underlying microscopic reasoning for the observed behavior. Given that the lattice constant for $\mathrm{EuO}(5.1435 \AA)$ [15] is larger than intermediate valence bulk $\mathrm{SmO}(4.9414 \AA)$ [14], one would expect that there is enough room to stabilize the 


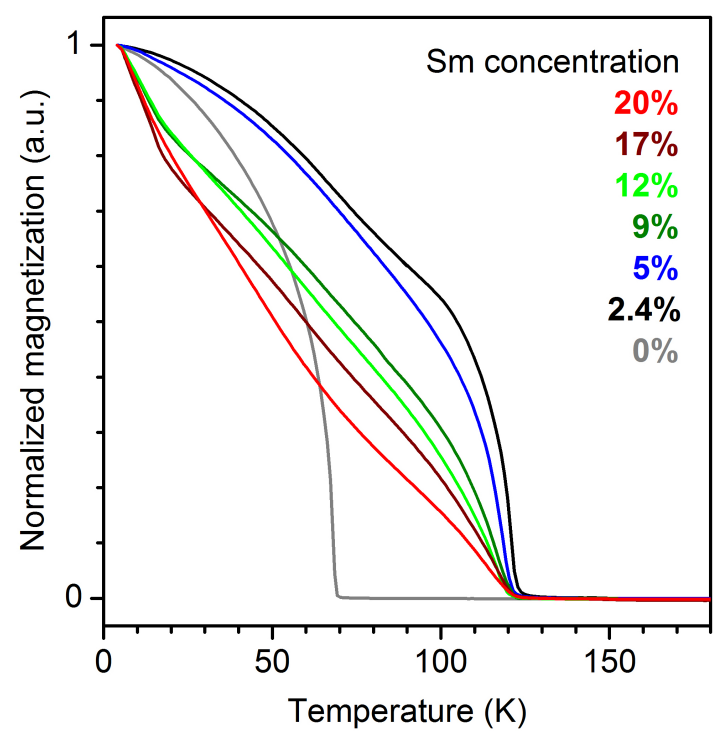

FIG. 6. Normalized temperature-dependent magnetization curves of the $\mathrm{Sm}_{\mathrm{x}} \mathrm{Eu}_{1-\mathrm{x}} \mathrm{O}$ films.

larger $\mathrm{Sm}^{2+}$ ions in $\mathrm{Sm}_{\mathrm{x}} \mathrm{Eu}_{1-\mathrm{x}} \mathrm{O}$. The observed unexpected $3+$ valency can be reasoned as follows. Comparing the lattice constants of $f c c-\mathrm{NiO}(4.17 \AA)$ [37] and $f c c-\mathrm{EuO}(5.1435 \AA)[15]$, one can infer that the larger lattice parameter of $\mathrm{EuO}$ originates from the fact that the ionic radius of $\mathrm{Eu}^{2+}$ is larger than both the $\mathrm{Ni}^{2+}$ and the $\mathrm{O}^{2-}$ and that it is the $\mathrm{Eu}^{2+}$ ions which determine the lattice constant. Thus, the $\mathrm{O}^{2-}$ ions have enough space to move or 'rattle' in this lattice. This should also be the case for $\mathrm{Sm}_{\mathrm{x}} \mathrm{Eu}_{1-\mathrm{x}} \mathrm{O}$, giving the possibility to gain energy by moving the $\mathrm{O}^{2-}$ ions closer to the Sm ions so as to form $\mathrm{Sm}^{3+}$ having the shorter Sm-O bonds. A similar observation was also made in $\mathrm{La}_{1.5} \mathrm{Sr}_{0.5} \mathrm{CoO}_{4}$, [38] in which both $\mathrm{Co}^{2+}$ and $\mathrm{Co}^{3+}$ ions occupy similar lattice sites. It was found that the $\mathrm{Co}^{3+}$ ions are in the low spin state although the lattice space would be sufficient to stabilize the larger ionic radius - high-spin $\mathrm{Co}^{3+}$ ions. [38]

\section{CONCLUSIONS}

We have prepared high quality single crystalline $\mathrm{Sm}_{\mathrm{x}} \mathrm{Eu}_{1-\mathrm{x}} \mathrm{O}$ thin films with $\mathrm{Sm}$ concentrations up to $20 \%$ by Eu distillation assisted MBE to study the valence state of Sm. Despite the large lattice spacing in the $\mathrm{EuO}$ rock salt crystal which is expected to support the divalent state of Sm, XPS and magnetization measurements reveal that the incorporated Sm ions exhibit a predominantly trivalent character. The Curie temperature of the Sm-doped $\mathrm{EuO}$ films can be enhanced up to $122 \mathrm{~K}$ confirming an effective transfer of charge from the $\mathrm{Sm}$ to the unoccupied states of the Eu ions. We infer that the large $\mathrm{EuO}$ lattice allows the oxygen ions to move locally in the structure, forming shorter Sm-O bonds and thereby stabilizing the $\mathrm{Sm}^{3+}$ valence.

\section{ACKNOWLEDGMENTS}

We thank Ch. Becker and T. Mende for their skillful technical assistance during experiments. The purification of the samarium by P. Höhn is gratefully acknowledged.
[1] M. Dzero, K. Sun, V. Galitski, and P. Coleman, Phys. Rev. Lett. 104, 106408 (2010).

[2] T. Takimoto, J. Phys. Soc. Jpn. 80, 123710 (2011).

[3] Z. Li, J. Li, P. Blaha, and N. Kioussis, Phys. Rev. B 89, 121117 (R) (2014).

[4] D. Kasinathan, K. Koepernik, L. H. Tjeng, and M. W. Haverkort, Phys. Rev. B 91, 195127 (2015).

[5] E. E. Vainshtein, S. M. Blokhin, and Y. B. Paderno, Sov. Phys. Solid State 6, 2318 (1965).

[6] A. Menth, E. Buehler, and T. H. Geballe, Phys. Rev. Lett. 22, 295 (1969).

[7] V. A. Sidorov, N. N. Stepanov, L. G. Khvostantsev, O. B. Tsiok, A. V. Golubkov, V. S. Oskotski, and I. A. Smirnov, Semicond. Sci. Technol. 4, 286 (1989).

[8] N. Yacoubi, J. M. Léger, and C. Loriers, J. de Physique Colloques 40, C5 (1979).

[9] P. Peratheepan and A. M. Strydom, J. Phys.: Condens. Matter 27, 095604 (2015).

[10] J. Arvanitidis, K. Papagelis, S. Margadonna, K. Prassides, and A. N. Fitch, Nature 425, 599 (2003).

[11] G. K. Wertheim and M. Campagna, Chem. Phys. Lett. 47, 182 (1977).
[12] R. Sutarto, S. G. Altendorf, B. Coloru, M. Moretti Sala, T. Haupricht, C. F. Chang, Z. Hu, C. SchüßlerLangeheine, N. Hollmann, H. Kierspel, H. H. Hsieh, H.J. Lin, C. T. Chen, and L. H. Tjeng, Phys. Rev. B 79, 205318 (2009).

[13] J. M. Leger, N. Yacoubi, and J. Loriers, Inorg. Chem. 19, 2252 (1980).

[14] G. Krill, M. F. Ravet, J. P. Kappler, L. Abadli, J. M. Leger, N. Yacoubi, and C. Loriers, Solid State Commun. 33, 351 (1980).

[15] O. D. McMasters, K. A. Gschneidner, E. Kaldis, and G. Sampietro, J. Chem. Thermodyn. 6, 845 (1974).

[16] P. G. Steeneken, L. H. Tjeng, I. Elfimov, G. A. Sawatzky, G. Ghiringhelli, N. B. Brookes, and D.-J. Huang, Phys. Rev. Lett. 88, 047201 (2002).

[17] A. Schmehl, V. Vaithyanathan, A. Herrnberger, S. Thiel, C. Richter, M. Liberati, T. Heeg, M. Röckerath, L. F. Kourkoutis, S. Mühlbauer, P. Böni, D. A. Muller, Y. Barash, J. Schubert, Y. Idzerda, J. Mannhart, and D. G. Schlom, Nat. Mater. 6, 882 (2007).

[18] J. B. Torrance, M. W. Shafer, and T. R. McGuire, Phys. Rev. Lett. 29, 1168 (1972). 
[19] Y. Shapira, S. Foner, and T. B. Reed, Phys. Rev. B 8, 2299 (1973).

[20] K. Y. Ahn and M. W. Shafer, J. Appl. Phys. 41, 1260 (1970).

[21] H. Y. Wang, J. Schoenes, and E. Kaldis, Helv. Phys. Acta 59, 102 (1986).

[22] F. Strisland, S. Raaen, A. Ramstad, and C. Berg, Phys. Rev. B 55, 1391 (1997).

[23] A. A. Samokhvalov, T. I. Arbuzova, V. S. Babushkin, B. A. Gizhevskii, N. N. Efremova, M. I. Simonova, and N. M. Chebotaev, Sov. Phys. Solid State 18, 1655 (1976).

[24] S. Hüfner, in Photoelectron Spectroscopy (Springer, 2003).

[25] G. K. Wertheim and G. Crecelius, Phys. Rev. Lett. 40, 813 (1978).

[26] E. L. Boyd, Phys. Rev. 145, 174 (1966).

[27] K. Y. Ahn and T. R. McGuire, J. Appl. Phys. 39, 5061 (1968).

[28] A. A. Samokhvalov, B. A. Gizhevskii, M. I. Simonova, and N. I. Solin, Sov. Phys. Solid State 14, 230 (1972).

[29] H. Ott, S. J. Heise, R. Sutarto, Z. Hu, C. F. Chang, H. H. Hsieh, H.-J. Lin, C. T. Chen, and L. H. Tjeng, Phys. Rev. B 73, 094407 (2006).

[30] R. Sutarto, S. G. Altendorf, B. Coloru, M. Moretti Sala, T. Haupricht, C. F. Chang, Z. Hu, C. SchüßlerLangeheine, N. Hollmann, H. Kierspel, J. A. Mydosh, H. H. Hsieh, H.-J. Lin, C. T. Chen, and L. H. Tjeng, Phys. Rev. B 80, 085308 (2009).
[31] A. Melville, T. Mairoser, A. Schmehl, D. E. Shai, E. J. Monkman, J. W. Harter, T. Heeg, B. Holländer, J. Schubert, K. M. Shen, J. Mannhart, and D. G. Schlom, Appl. Phys. Lett. 100, 222101 (2012).

[32] H. Miyazaki, H. J. Im, K. Terashima, S. Yagi, M. Kato, K. Soda, T. Ito, and S. Kimura, Appl. Phys. Lett. 96, 232503 (2010).

[33] S. G. Altendorf, A. Reisner, C. F. Chang, N. Hollmann, A. D. Rata, and L. H. Tjeng, Appl. Phys. Lett. 104, 052403 (2014).

[34] L. D. Finkelstein, N. N. Efremova, M. I. Simonova, N. I. Lobachevskaya, A. A. Samokhvalov, V. G. Bamburov, and S. A. Nemnonov, Sov. Phys. Solid State 19, 2168 (1977).

[35] A. A. Samokhvalov, B. A. Gizhevskii, M. I. Simonova, N. M. Chebotaev, and L. D. Falkovskaya, Sov. Phys. Solid State 24, 1103 (1982).

[36] S. G. Altendorf, N. Hollmann, R. Sutarto, C. Caspers, R. C. Wicks, Y.-Y. Chin, Z. Hu, H. Kierspel, I. S. Elfimov, H. H. Hsieh, H.-J. Lin, C. T. Chen, and L. H. Tjeng, Phys. Rev. B 85, 081201 (2012).

[37] S. J. Bhatt and H. D. Merchant, J. Am. Ceram. Soc. 52, 452 (1969).

[38] C. F. Chang, Z. Hu, H. Wu, T. Burnus, N. Hollmann, M. Benomar, T. Lorenz, A. Tanaka, H.-J. Lin, H. H. Hsieh, C. T. Chen, and L. H. Tjeng, Phys. Rev. Lett. 102, 116401 (2009). 